\title{
The difference in clinical characteristics in the patients with unilateral oral cenesthopathy with and without the neurovascular compression of trigeminal nerve: A clinical chart review
}

\section{Kazuya Watanabe}

Tokyo Medical and Dental University

Motoko Watanabe ( $\nabla$ totoompm@tmd.ac.jp)

Tokyo Medical and Dental University

\section{Takayuki Suga}

Tokyo Medical and Dental University

Trang Thi Huyen Tu

Tokyo Medical and Dental University

Chaoli Hong

Tokyo Medical and Dental University

Zhenyan Liu

Tokyo Medical and Dental University

Chihiro Takao

Tokyo Medical and Dental University

Junichiro Sakamoto

Tokyo Medical and Dental University

Norio Yoshino

Tokyo Medical and Dental University

\section{Yojiro Umezaki}

Fukuoka Shika Daigaku

\section{Tatsuya Yoshikawa}

Tokyo Medical and Dental University

Miho Takenoshita

Tokyo Medical and Dental University

Haruhiko Motomura

Tokyo Medical and Dental University

Tohoru Kurabayashi

Tokyo Medical and Dental University

Akira Toyofuku

Tokyo Medical and Dental University 


\section{Research article}

Keywords: Oral cenesthopathy, Neurovascular compression, Oral somatic delusion, Trigeminal nerves, Oral DRS

Posted Date: June 19th, 2020

DOI: https://doi.org/10.21203/rs.3.rs-33642/v1

License: (c) (1) This work is licensed under a Creative Commons Attribution 4.0 International License. Read Full License 


\section{Abstract \\ Background}

Oral cenesthopathy $(\mathrm{OC})$ is characterized by unusual sensation in the oral cavity without corresponding medical and dental evidence. Their complaints are vary and some patients show sever symptoms which decline their quality of life. The neurovascular compression (NVC) of trigeminal nerves is known as one of the factors to indicate abnormal sensation in facial area including oral cavity. The aim of the present study was to investigate the pathophysiology of $\mathrm{OC}$ by comparing the clinical characteristics of unilateral OC patients with and without trigeminal nerve NVC.

\section{Methods}

This is a retrospective study reviewing medical records of patients diagnosed with unilateral OC seen between April 2016 and February 2019. Magnetic resonance images (MRI) were performed to assess the presence of NVC.

\section{Results}

Among 321 patients who were diagnosed with OC, 87 patients showed unilateral OC. finally, 52 patients who were performed MRI were involved in the present investigation. In $48.1 \%$ subjects $(25 / 52)$, NVC of trigeminal nerves was observed. In the patients without NVC, $85.2 \%(23 / 27)$ were female with significant difference $(p=0.010)$ compared to the patients with NVC $(48.0 \%, 12 / 25)$. There was no significant difference in age, the presence of psychiatric history and psychosomatic dental comorbidity, and the scores of psychological questionnaires. However, in the patients without NVC, significantly higher scores were observed in the symptom severity and the functional impairment as follow; movement $(p=0.042)$, pain $(p=0.040)$, work $(p=0.013)$ and social activities $(p=0.015)$, compared to the group with NVC.

\section{Conclusion}

These results suggest that unilateral $\mathrm{OC}$ has two subtypes based on predominant involvement of peripheral or central nervous system (CNS). The unilateral OC with NVC may involve predominantly peripheral nervous system. Furthermore, the unilateral OC without trigeminal nerve NVC which showed severe functional impairment may be reflected predominant CNS involvement.

\section{Background}

Oral cenesthopathy $(\mathrm{OC})$ is characterized by unusual sensations in the oral cavity without corresponding medical and dental evidence. OC is also called "oral somatic delusion" or "oral dysesthesia". OC patients complaint various sensations. Some patients complaint of a slimy and sticky sensation or excessive 
mucus secretion in the oral cavity while some patients complain of real strange oral sensations such as coil and wire sometimes even accompanied by the movement. Their daily lives are sometimes eclipsed by bizarre symptoms. It remains still unclear the detailed pathophysiology of $\mathrm{OC}$ including the factors which affect variations and severities of symptoms.

OC is classified as "delusional disorder, somatic form" according to the Diagnostic and Statistical Manual of Mental Disorders, 5th Edition [1], and classified as "persistent delusional disorder" or "other schizophrenia" in the classification of mental and behavioral disorders in International Classification of Diseases 10th revision [2]. Previously, the existence of the subtypes of OC have been suggested based on the etiology [3] and clinical mental manifestation [4]. Moreover, since the comorbidity of psychotic disorders such as schizophrenia and bipolar was found only $6 \%$ in the OC patients, the authors conclude the oral cenesthopathic sensation might not be merely a partial symptom of psychotic disorders that present delusional symptoms [5]. These results suggest that the pathophysiology of OC differ from psychotic disorders.

Recently, involvement of the central nervous system (CNS) was considered as one of the pathophysiology of OC. In the investigation by using single photon emission computed tomography (SPECT), cerebral blood flow (CBF) showed a right-dominant asymmetry in the callosomarginal, precentral, and temporal regions in the patients with $\mathrm{OC}$ [6]. Moreover, a significant right dominant asymmetry in the temporal and posterior cerebral regions was observed regardless of the presence of a history of depression [7]. The right dominant asymmetric CBF may affect the oral delusional symptoms.

As the peripheral nervous system, cranial nerves assume important roles. The trigeminal nerve is the largest cranial nerve and has a sensitive and motor composition for a facial area including oral cavity. It is called root entry zone (REZ) where trigeminal nerves entry into or exit from brainstem via the cisternal portion at the height of pons. The artery or vein compression of trigeminal nerves can be occurred at REZ. Neurovascular compression (NVC) of trigeminal nerves indicates abnormal pain sensation such as trigeminal neuralgia [8], persist idiopathic facial pain (PIFP) in some cases [9]. Since trigeminal nerves transit not only pain but also thermal, tactile and pressure, we hypothesized that NVC of trigeminal nerves may be involved in the pathophysiology of other abnormal oral sensation in $\mathrm{OC}$ patients. The trigeminal nerves mainly decussates and run to contralateral thalamic neuclei which project to cortical region through the thalamocortical tract [10]. Considering this anatomical nerve tract and asymmetric CBF in OC patients, other our hypothesis was built that unilateral OC has subtypes based on not only the presence of NVC but also the laterality of the symptoms and NVC.

The aim of the present study was to investigate the pathophysiology of OC by comparing clinical characteristics of unilateral OC patients with and without NVC of trigeminal nerves.

\section{Methods}

\section{Subjects}


This is a retrospective study reviewing the medical records of patients diagnosed with unilateral OC seen between April 2016 and February 2019 at the Psychosomatic Dentistry Clinic of Tokyo Medical and Dental University.

The OC was diagnosed by a specialist in psychosomatic dentistry who had certified by Japanese society of psychosomatic dentistry. The inclusion criteria were as follows: 1) unilateral symptoms of OC, 2) absence of corresponding abnormal findings from a dental panoramic radiograph and intra- and extraoral examination. The exclusion criteria were patients who 1) did not give us informed consent, 2) referred to another clinic, 3) dropped out, 4) refused inspection, 5) could not take magnetic resonance images (MRI), 6) had a severe health condition and 7) were performed MRI in the another year during the study duration (Fig. 1).

Clinical characteristics were obtained from the patients' medical charts, including demographic information (sex, age), the duration of illness, psychiatric history, comorbid psychosomatic dental symptoms, and the triggers of symptoms. All examiners in the present study were well experienced trained clinicians and researchers in our clinic.

\section{MRI protocol and definitions}

All patients underwent MRI scan within one month after the first medical examination. No patients had improved their oral symptoms on that day. All data were acquired on 3-Tesla 3D MRI scanner (Siemens Healthcare, Erlangen, Germany) with a 16-channel head coil. The MRI sequences were acquired at the level of REZ of trigeminal nerves with the same parameters with our previous study [9]. MR angiography (MRA) was acquired using 3D time-of-flight (3D-TOF) MRA with following parameters: repetition time (TR) / echo time (TE) $24 / 3.9 \mathrm{~ms}$, flip angle $18^{\circ}$, field of view (FOV) $160 \times 160 \mathrm{~mm}$, matrix $320 \times 192$, section thickness $0.5 \mathrm{~mm}$, and number of slab 3 , which was reconstructed to a voxel size of $0.5 \times 0.5 \times$ $0.5 \mathrm{~mm}$, a slab thickness $44 \mathrm{~mm}$. MR cisternography was obtained using 3D constructive interference in steady state (3D-CISS) with the following parameter: TR/TE 7.4/3.7 ms, flip angle 50 ${ }^{\circ}$, FOV $160 \times$ $160 \mathrm{~mm}$, matrix $320 \times 320$, section thickness $0.5 \mathrm{~mm}$, which was reconstructed to a voxel size of $0.5 \times 0.5$ $\times 0.5 \mathrm{~mm}$, a slab thickness $44 \mathrm{~mm}$.

All 3D-TOF and 3D-CISS were displayed in triplanar views (transverse, coronal and sagittal views) on the visualization system and evaluated the presence of NVC by experienced oral radiologists who were blinded to symptom side. The presence of NVC was defined as contact between a blood vessel and trigeminal nerves at REZ without visible cerebrospinal fluid between them in the triplanar views of 3DCISS. The type of responsible blood vessel (artery or vein) was evaluated using the triplanar views and memory-in-pixel display of 3D-TOF MRA. If there was disagreement or uncertainty about whether there was a contact, it was considered as no NVC in the data analysis.

\section{Assessment of $\mathrm{OC}$ and psychological state}

The symptoms of OC was assessed using the oral dysesthesia rating scale (Oral DRS) which developed based on literature review and extensive clinical experience to reorganize and objectify the complicate 
symptoms [11]. Oral DRS consists of a symptom severity scale (SSS), functional impairment scale (FIS), and visual analog scale (VAS). In the SSS [A], oral symptoms are classified into seven categories: feelings of foreign body [A1], exudation [A2], squeezing pulling [A3], movement [A4], misalignment [A5], pain [A6], and spontaneous thermal sensation or tastes [A7]. The FIS [B] assesses the degree of impairment of eating [B1], articulation [B2], work [B3], and social activities [B4]. The VAS [C] consists of two scales that assess overall subjective severity of the symptoms [C1] and changes in severity of the symptoms [C2]. The Oral DRS is available in Japanese and English with the respective instruction manuals on the website: http://www.tmd.ac.jp/grad/ompm/details8.html.

Two questionnaires were conducted to assess mental and psychological condition. Zung's self-rating depression scale (SDS) was performed to assess the depression status [12][13] and the short intolerance of uncertainty scale (SIUS) was performed to assess in the tendency of uncertainty intolerance [14][15]. For the assessment of comorbidities of functional somatic symptoms, somatic symptom scale 8 (SSS-8) was implemented. The eight descriptors contained SSS-8 were rated and used to evaluate the mental and psychological effects on functional somatic symptoms [16][17].

Pain catastrophizing scale (PCS) used to evaluate pain catastrophizing [18][19]. Subgrouping for targeted treatment generic (STarT-G) were also performed to assess disability due to chronic sensation [20]. In addition, clinical global impression (CGI) severity was used for objective assessment of the severity of illness [21].

\section{Analysis}

All data were analyzed using student t-test, Mann-Whitney U test and Fisher's exact test. The student ttest (age and the duration of illness), Mann-Whitney $U$ test (CGI), and Fisher's exact test (the other demographic and clinical characteristics) was used to compare between the patients with trigeminal NVC and the patients without it. The student's t-test was used to compare the scores of SDS, SSS-8, SIUS, PCS and STarT-G between two groups. The score of Oral DRS were compared by using Mann-Whitney Utest. All analyses were performed with the statistical software package $\mathrm{R}$ for Mac, version $\mathrm{R}$ Ver 2.2.1. with the statistical significance $(p<0.05)$.

\section{Result}

\section{Study subjects}

From April 2016 to February 2019, 1478 patients visited to the psychosomatic dentistry clinic of Tokyo Medical and Dental University Hospital. Among them, 321 patients were diagnosed with OC and unilateral OC was observed in 87 patients. Except for 35 patients who satisfied exclusion criteria, 52 patients were performed MRI and assessed the presence of NVC (Fig. 1).

\section{Demographic and clinical characteristics}


The NVC on the trigeminal nerves were observed in 25 patients (48.1\%) with unilateral OC (Table 1). One case which observed NVC of the trigeminal nerves was shown in Fig. 2. In the patients without NVC, the rate of female was $85.2 \%(23 / 27)$ and significantly high $(p=0.010)$ compared to that of the patients with NVC $48.0 \%(12 / 25)$. For the side of symptom, 74.1\% (20/27) patients without NVC showed the symptoms in their left, but no significance was found regardless the presence of NVC $(p=0.627)$. In the patients with NVC, $52.0 \%$ were found ipsilaterally, $32.0 \%$ were contralateral, and $16.0 \%$ were bilateral. There was no significant difference in age, the presence of psychiatric history and comorbid psychosomatic dental symptoms, the trigger of the symptoms, duration of illness and CGI severity.

\section{Comparisons of questionnaires}

No significant difference was found in SDS, PCS, SSS-8, SIUS, and STarT-G compared between groups with or without NVC (Table 2). However, the patients without NVC showed significantly higher VAS and the total scores of the symptom severity and the functional impairment in Oral DRS $(46.1 \pm 8.3 ; p=0.016$, $13.2 \pm 8.3 ; p=0.012,6.7 \pm 4.1 ; p=0.022$, respectively), compared to the patients with NVC. For the details, the scores in movement $(p=0.042)$, pain $(p=0.040)$, work $(p=0.006)$ and social activities $(p=0.015)$ were significant (Fig. 3). Moreover, the numbers of corresponding symptoms in both SSS and FIS were significantly higher in the patients without NVC than those in the patients with NVC.

\section{Discussion}

This is the first study investigated pathophysiological involvement of NVC of trigeminal nerves by comparing the clinical characteristics of unilateral OC patients with and without trigeminal NVC. The NVC of trigeminal nerves were observed in $48.1 \%$ patients, and the patients without NVC were found predominantly female and showed more complex symptoms and sever dysfunction in their daily lives.

OC patients complain of various discomfort or abnormal sensations in the oral cavity without dental and medical evidence. Some complaints are on the extension of understandable sensations such as sticky or slimy sensation, but some are more bizarre and realistic feeling as if wires, coil, small bubbles or eggs exist even the patients recognized they do not. The severity of the symptoms is also various. In some patients, their daily lives are eclipsed by the oral symptoms and their quality of life are declined. Several studies have proposed etiological hypotheses to explain clinical features and treatment effects.

The CNS involvement were suggested in previously. In a case report with OC, the asymmetric CBF pattern ameliorated after the treatment with aripiprazole [22]. The case report of the modified electroconvulsive therapy to the $\mathrm{OC}$ patients showed improved CBF asymmetry in the temporal region and reduced the symptoms of OC [23]. In addition, the investigation by using SPECT also revealed that CNS might be involved. The right-dominant regional CBF ( $\mathrm{CCBF}$ ) asymmetry was observed in the callosomarginal, precentral, and temporal regions in the patients with OC [6]. Moreover, a significant right dominant asymmetry in the temporal and posterior cerebral regions was observed regardless of the presence of a 
history of depression, while the mean rCBF values of patients with depression were lower in several regions [7].

To the peripheral, trigeminal nerve is the fifth and the largest cranial nerve. It is composed of sensory and motor nerves extensively distribute to facial area including oral cavity. The NVC at the REZ of trigeminal nerves indicates abnormal pain sensation such as trigeminal neuralgia, PIFP. Since trigeminal nerves transit not only pain but also thermal, tactile and pressure, there is possibility that NVC of trigeminal nerves also indicate abnormal sensation which found in OC patients. In the present study, 48.1\% of the unilateral OC patients showed NVC and the rate was similar with our previous study of the unilateral PIFP patients [9]. The patients without NVC of trigeminal nerves was found in $51.9 \%$ patients and predominantly female. The rate (85.2\%) coincides with the previous studies of OC (73.8\%) [5] and other psychosomatic dental symptoms [24][25][26]. Moreover, the patients without NVC showed significantly more complexed oral symptoms with higher severity and functional impairment in working and social activity which decline the quality of life though no significance were observed in psychological questioners compared to the patient with NVC. These results suggest that the unilateral OC has two subtypes based on predominant involvement of central or peripheral nervous system. In speculate, the more complex mechanisms which involved in CNS may exist for the cases without NVC and may indicate various and severer symptoms. Additionally, some mechanisms which modulate the sensation may exist for the case with NVC.

The myelin sheath surrounding the cranial nerves to protect from damage and support metabolism. In the CNS, oligodendrocytes form the myelin while Schwann cell form in the peripheral nervous system [27]. The transit zone of these two types of myelin is more vulnerable. The chronic demyelination and aberrant re-myelination at the transit zone were noticed as the underling mechanism involved in the syndrome caused by NVC [8]. Interestingly, the myelin sheath of demyelinated and abnormally remyelinated were as thin as normal white matter [28], and uncertain degeneration case reduction of nerve conduction velocity [29]. The neural inflammation, edema and plasticity on the trigeminal nerves indicate the white matter abnormalities in the brainstem project to somatosensory cortices [10]. While these abnormalities may occur idiopathic sensation in facial area, is there any possibility to speculate that these degenerative changes effect to control the sensation of OC not to exacerbate.

In addition, most patients with trigeminal neuralgia complain unilateral symptoms, but the laterality, whether left or right, is not significant [30]. On the other hand, 234/321 OC patients showed bilateral symptoms who were excluded from the present study, and left side unilateral symptoms were found in $69.2 \%$ patients with unilateral OC. Although no significant was found in this study due to the small sample size, the left dominant symptom laterality and the ipsilateral NVC would be key points for the next investigations considering right dominant asymmetric CBF in the patients with OC [6][7]. Further studies combined with CNS and peripheral nervous system were required to investigate highly complexed pathophysiology of OC.

\section{Limitations}


The present study has several limitations. First, the MRI scan were preformed within one month from the first visit, and some patients had already underwent the psychopharmaco-treatment. However, there was no patient whose symptom had improved on the day of the MRI examination. Second, the comparison with the state after the treatment was not investigated. To compare them, further detailed involvement of NVC of trigeminal nerves will be revealed in the future. Third, the examination to assess the involvement of CNS were not performed. Besides SPECT, diffusion tensor imaging is also useful to investigate nerve fibers connectivity in both peripheral and central nervous systems [30].

\section{Conclusion}

In the present study, the patients without NVC were found predominantly female and showed significantly higher severity and dysfunction in their daily activities compared to the patients with NVC. These results suggest that unilateral $\mathrm{OC}$ has two subtypes based on predominant involvement of peripheral or central nervous system. The unilateral OC with NVC may involve predominantly peripheral nervous system. Furthermore, the unilateral OC without trigeminal nerve NVC which showed severe functional impairment may be reflected predominant CNS involvement.

\section{Abbreviations}

OC, Oral cenesthopathy; NVC, neurovascular compression; CNS, central nervous system; SPECT, single photon emission computed tomography; $\mathrm{CBF}$, cerebral blood flow; REZ, root entry zone; MRI, magnetic resonance images; MRA, MR angiography; 3D-TOF, 3D time-of-flight; TR, repetition time; TE, echo time; FOV, field of view; 3D-CISS, 3D constructive interference in steady state; Oral DRS, oral dysesthesia rating scale; SSS, symptom severity scale; FIS, functional impairment scale; VAS, visual analog scale; SDS, Zung's self-rating depression scale; SIUS, short intolerance of uncertainty scale; SSS-8, somatic symptom scale 8; PCS, Pain catastrophizing scale; STarT-G, Subgrouping for targeted treatment generic; $\mathrm{CGI}$, clinical global impression; rCBF, regional CBF

\section{Declarations}

\section{Ethics approval and consent to participate}

This study is approved by the Ethical Committee of Tokyo Medical and Dental University Dental Hospital (approval number: D2013-005). All patients provided written informed consent.

\section{Consent for publication}

Consent for publication were obtained from all patients.

\section{Availability of data and materials}


The datasets used and analyzed during the present study are available from the corresponding author on reasonable request.

\section{Competing interests}

The authors declare that they have no competing interests.

\section{Funding}

The present study was supported by KAKENHI from Japanese Society for the Promotion of Science (JSPS), Grant Number 19K10328 to Prof. Toyofuku. The funder had no role in study design, data collecting, data analysis, decision of publishing and preparation of the manuscript.

\section{Authors' contribution}

KW acquired and analyzed the data and wrote the first draft of the manuscript. TS, TT, CH, ZL, CT, TY and MT acquired the data. JS, NY and TK acquired and analyzed the imaging data. MW, YU, HM analyzed the data and revised the manuscript. AT was contributed to study design and developed the methodology. All authors read and approved the final manuscripts.

\section{Acknowledgements}

We thank for all staff and technicians (Department of Oral and Maxillofacial Radiology, Graduate school of Medical and Dental University) for their help to perform MRI examinations.

\section{References}

1. American Psychiatric Association. Diagnostic and Statistical Manual of Mental Disorders. 5th ed. American P. Washington, DC; 2013.

https://dsm.psychiatryonline.org/doi/book/10.1176/appi.books.97808904255966. Accessed September 24, 2018.

2. World Health Organization. The ICD-10 classification of mental and behavioural disorders. clinical descriptions and diagnostic guidelines. Geneva: World Health Organization; 1992.

3. Hozaki H. Cenestopathia and related condition. Seishin Igaku (in Japanese). 1960;2:325-32.

4. Yoshimatsu K. The psychopathology of cenesthopsthia. Seishinshinkeigaku zasshi (in Japanese). 1996;12:347-55.

5. Umezaki Y, Miura A, Shinohara Y, Mikuzuki L, Sugawara S, Kawasaki K, et al. Clinical characteristics and course of oral somatic delusions: a retrospective chart review of 606 cases in 5 years. Neuropsychiatr Dis Treat. 2018;14:2057-65. doi:10.2147/NDT.S167527.

6. Umezaki Y, Katagiri A, Watanabe M, Takenoshita M, Sakuma T, Sako E, et al. Brain perfusion asymmetry in patients with oral somatic delusions. Eur Arch Psychiatry Clin Neurosci.

2013;263:315-23. 
7. Watanabe M, Umezaki Y, Miura A, Shinohara Y, Yoshikawa T, Sakuma T, et al. Comparison of cerebral blood flow in oral somatic delusion in patients with and without a history of depression: A comparative case series. BMC Psychiatry. 2015;15:42.

8. Love S. Trigeminal neuralgia: Pathology and pathogenesis. Brain. 2001;124:2347-60.

9. Kawasaki K, Sugawara S, Watanabe K, Hong C, Tu TTH, Watanabe T, et al. Differences in the Clinical Characteristics of Persistent Idiopathic Facial Pain (Atypical Odontalgia) Patients with or Without Neurovascular Compression of the Trigeminal Nerve. Pain Med. 2020;21:814-21.

10. Moayedi M, Hodaie M. Trigeminal nerve and white matter brain abnormalities in chronic orofacial pain disorders. PAIN Reports. 2019;4:e755.

11. Uezato A, Toyofuku A, Umezaki Y, Watanabe M, Toriihara A, Tomita M, et al. Oral Dysesthesia Rating Scale: A tool for assessing psychosomatic symptoms in oral regions. BMC Psychiatry. 2014;14:1696.

12. ZUNG WW. A SELF-RATING DEPRESSION SCALE. Arch Gen Psychiatry. 1965;12:63-70. doi:10.1001/archpsyc.1965.01720310065008.

13. Gormsen L, Rosenberg R, Bach FW, Jensen TS. Depression, anxiety, health-related quality of life and pain in patients with chronic fibromyalgia and neuropathic pain. Eur J Pain. 2010;14:127.e1-127.e8.

14. Boelen PA, Vrinssen I, van Tulder F. Intolerance of uncertainty in adolescents: correlations with worry, social anxiety, and depression. J Nerv Ment Dis. 2010;198:194-200.

doi:10.1097/NMD.0b013e3181d143de.

15. Bélanger C, Blais Morin B, Brousseau A, Gagné N, Tremblay A, Daigle K, et al. Unpredictable pain timings lead to greater pain when people are highly intolerant of uncertainty. Scand J Pain. 2017;17:367-72.

16. Gierk B, KohImann S, Kroenke K, Spangenberg L, Zenger M, Brahler E, et al. The Somatic Symptom Scale-8 (SSS-8): A brief measure of somatic symptom burden. JAMA Intern Med. 2014;174:399-407.

17. Matsudaira K, Oka H, Kawaguchi M, Murakami M, Fukudo S, Hashizume M, et al. Development of a Japanese version of the Somatic Symptom Scale-8: Psychometric validity and internal consistency. Gen Hosp Psychiatry. 2017;45:7-11.

18. Sullivan MJL, Bishop SR, Pivik J. The Pain Catastrophizing Scale: Development and Validation. Psychol Assess. 1995;7:524-32.

19. Quartana PJ, Campbell CM, Edwards RR. Pain catastrophizing a critical review. Expert Rev Neurother. 2009;9:745-58.

20. Oka H, Matsudaira K, Fujii T, Kikuchi N, Haga Y, Sawada T, et al. Estimated risk for chronic pain determined using the generic STarT Back 5-item screening tool. J Pain Res. 2017;10:461-7.

21. Guy W. ECDEU Assessment Manual for Psychopharmacology. Rockvill, MD: US Department of Health, Education and Welfare Public Health Service Alcohol, Drug Abuse, and Mental Health Administration; 1976.

22. Umezaki Y, Uezato A, Toriihara A, Nishikawa T, Toyofuku A. Two cases of oral somatic delusions ameliorated with brain perfusion asymmetry: A case report. Clin Neuropharmacol. 2017;40:97-9. 
23. Uezato A, Yamamoto N, Kurumaji A, Toriihara A, Umezaki Y, Toyofuku A, et al. Improvement of asymmetrical temporal blood flow in refractory oral somatic delusion after successful electroconvulsive therapy. J ECT. 2012;28:50-1. doi:10.1097/YCT.0b013e31822e581e.

24. Savage NW. Burning mouth syndrome: patient management. Aust Dent J. 1996;41:363-6. doi:10.1111/j.1834-7819.1996.tb06019.x.

25. Tu TTH, Miura A, Shinohara Y, Mikuzuki L, Kawasaki K, Sugawara S, et al. Pharmacotherapeutic outcomes in atypical odontalgia: determinants of pain relief. J Pain Res. 2019;12:831-9. doi:10.2147/JPR.S188362.

26. Tu TTH, Takenoshita M, Matsuoka H, Watanabe T, Suga T, Aota $Y$, et al. Current management strategies for the pain of elderly patients with burning mouth syndrome: a critical review. Biopsychosoc Med. 2019;13:1. doi:10.1186/s13030-019-0142-7.

27. Haller S, Etienne L, Kövari E, Varoquaux AD, Urbach H, Becker M. Imaging of Neurovascular Compression Syndromes: Trigeminal Neuralgia, Hemifacial Spasm, Vestibular Paroxysmia, and Glossopharyngeal Neuralgia. AJNR Am J Neuroradiol. 2016;37:1384-92. doi:10.3174/ajnr.A4683.

28. Love S, Hilton DA, Coakham HB. Central Demyelination of the Vth Nerve Root in Trigeminal Neuralgia Associated with Vascular Compression. Brain Pathol. 2006;8:1-11.

29. Smith KJ, Hall SM. Nerve conduction during peripheral demyelination and remyelination. J Neurol Sci. 1980;48:201-19.

30. Ko AL, Lee A, Raslan AM, Ozpinar A, McCartney S, Burchiel KJ. Trigeminal neuralgia without neurovascular compression presents earlier than trigeminal neuralgia with neurovascular compression. J Neurosurg. 2015;123:1519-27.

\section{Tables}

\section{Table 1.}

Demographic and clinical characteristic of patients with unilateral oral cenesthopaty 


\begin{tabular}{|c|c|c|c|c|}
\hline & Total & Without NVC & With NVC & \multirow[t]{2}{*}{$p$-value } \\
\hline & $(n=52)$ & $\begin{array}{l}(n=27 \\
51.9 \%)\end{array}$ & $\begin{array}{l}(n=25 \\
48.1 \%)\end{array}$ & \\
\hline Female (\%) & 35 (67.3) & $23(85.2)$ & $12(0.48)$ & 0.010 * \\
\hline Age (mean \pm SD) & $\begin{array}{l}63.2 \pm \\
12.3\end{array}$ & $63.3 \pm 13.4$ & $63.0 \pm 11.4$ & 0.914 \\
\hline $\begin{array}{l}\text { Duration of illness (mean } \pm S D \text {, } \\
\text { months) }\end{array}$ & $\begin{array}{l}47.1 \pm \\
51.2\end{array}$ & $54.4 \pm 59.2$ & $39.2 \pm 40.6$ & 0.283 \\
\hline CGI (median) & 4 & 4 & 4 & 0.501 \\
\hline The side of symptom & & & & 0.627 \\
\hline Left (\%) & $36(69.2)$ & $20(74.1)$ & $16(64.0)$ & \\
\hline Right (\%) & $16(30.8)$ & $7(25.9)$ & $9(36.0)$ & \\
\hline \multicolumn{5}{|l|}{ Laterality of NVC } \\
\hline Ipsilateral (\%) & $13(25.0)$ & $0(0.0)$ & $13(52.0)$ & \\
\hline Contralateral (\%) & $8(15.4)$ & $0(0.0)$ & $8(32.0)$ & \\
\hline Bilateral (\%) & $4(7.7)$ & $0(0.0)$ & $4(16.0)$ & \\
\hline \multicolumn{5}{|l|}{ Psychiatric history } \\
\hline None (\%) & $17(32.7)$ & $8(29.6)$ & $9(36.0)$ & 0.662 \\
\hline Schizophrenia & 1 & 0 & 1 & \\
\hline Bipolar disorder & 4 & 3 & 1 & \\
\hline Depression & 12 & 6 & 6 & \\
\hline Anxiety disorder & 12 & 7 & 5 & \\
\hline Insomnia & 2 & 0 & 1 & \\
\hline Other & 1 & 1 & 0 & \\
\hline Detail is unknown & 3 & 2 & 1 & \\
\hline \multicolumn{5}{|l|}{$\begin{array}{l}\text { Comorbid psychosomatic dental } \\
\text { symptom }\end{array}$} \\
\hline None (\%) & $33(63.5)$ & $14(51.9)$ & $19(76.0)$ & 0.089 \\
\hline Burning mouth syndrome & 7 & 3 & 4 & \\
\hline Atypical odontalgia & 9 & 9 & 0 & \\
\hline
\end{tabular}




\begin{tabular}{|lllll|}
\hline Phantom bite syndrome & 1 & 1 & 0 \\
\hline Others & 3 & 1 & 2 & 0.588 \\
\hline Dental trigger of symptoms & & & & $10(40.0)$ \\
\hline None (\%) & $23(44.2)$ & $13(48.1)$ & 5 \\
\hline Dental caries treatment & 10 & 5 & 3 \\
\hline Tooth extraction & 6 & 3 & 3 \\
\hline Prosthodontic treatment & 7 & 4 & 1 \\
\hline Periodontal treatment & 2 & 1 & 1 \\
\hline Denture adjustment & 2 & 1 & 1 \\
\hline Occlusal adjustment & 1 & 0 & 1 \\
\hline Implant placement & 1 & 0 & \\
\hline
\end{tabular}

NVC; neurovascular compression

SD; standard deviasion

CGl; clinical glabal impression

$*: p<0.05$

\section{Table 2.}

Comparisons of questionnaires between the patients with and without NVC 


\begin{tabular}{|c|c|c|c|c|c|}
\hline Questionnaires (mean \pm SD) & Total & Without NVC & With NVC & $p$ value & \\
\hline VAS & $34.6 \pm 36.3$ & $46.1 \pm 36.0$ & $22.2 \pm 33.0$ & 0.016 & * \\
\hline SDS & $46.2 \pm 10.1$ & $47.0 \pm 10.1$ & $45.2 \pm 10.2$ & 0.536 & \\
\hline PCS & $28.4 \pm 15.4$ & $31.7 \pm 12.6$ & $24.8 \pm 17.6$ & 0.106 & \\
\hline SSS-8 & $6.9 \pm 4.1$ & $7.4 \pm 3.8$ & $6.4 \pm 4.5$ & 0.384 & \\
\hline SIUS & $30.6 \pm 10.2$ & $30.9 \pm 10.1$ & $30.2 \pm 10.4$ & 0.811 & \\
\hline STarT-G & $2.2 \pm 1.4$ & $2.3 \pm 1.2$ & $2.0 \pm 1.6$ & 0.461 & \\
\hline \multicolumn{6}{|l|}{ Oral DRS } \\
\hline SSS total & $10.9 \pm 6.9$ & $13.2 \pm 8.3$ & $8.5 \pm 3.8$ & 0.012 & * \\
\hline $\begin{array}{l}\text { The number of corresponding } \\
\text { symptoms in SSS }\end{array}$ & $3.8 \pm 1.8$ & $4.3 \pm 1.9$ & $3.2 \pm 1.5$ & 0.032 & * \\
\hline FIS total & $5.3 \pm 4.7$ & $6.7 \pm 4.1$ & $3.7 \pm 4.8$ & 0.022 & * \\
\hline $\begin{array}{l}\text { The number of corresponding } \\
\text { impairment in FIS }\end{array}$ & $2.3 \pm 1.4$ & $2.8 \pm 1.2$ & $1.7 \pm 1.4$ & 0.004 & * \\
\hline
\end{tabular}

NVC; neurovascular compression

SD; standard deviasion

VAS; visual analogue scale

SDS; Zung's self-rating depression scale

PCS; pain catastrophizing scale

SSS-8; somatic symptom scale 8

SIUS; short intolerance of uncertainty scale

STarT-G; subgrouping for targeted treatment generic

SSS; symptom severity scale

FIS; functional impairment scale

$* ; p<0.05$

Figures 


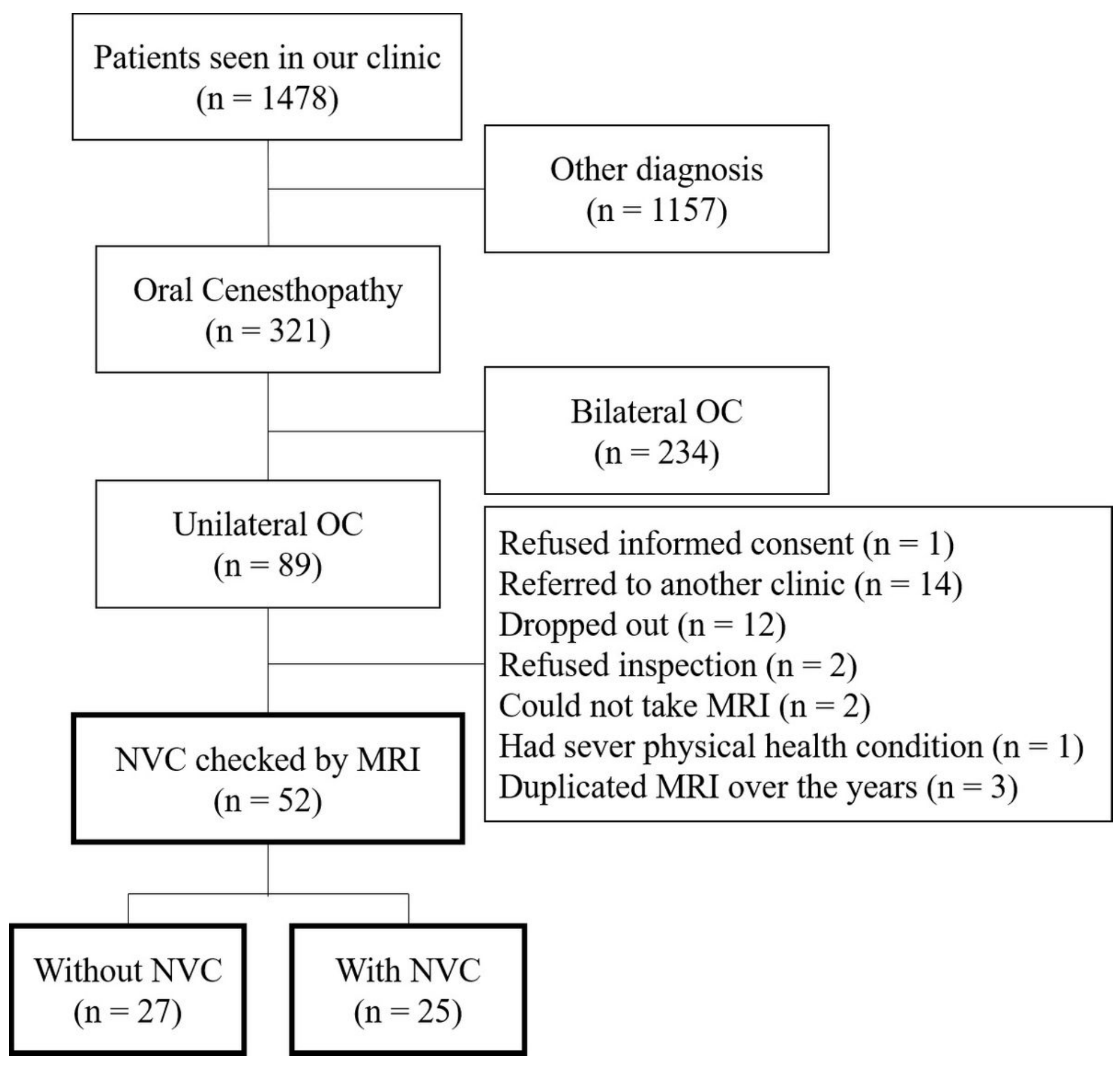

Figure 1

Flowchart showing the selection of patients with unilateral Oral Cenesthopathy for inclusion in the present study. 




\section{(B)}
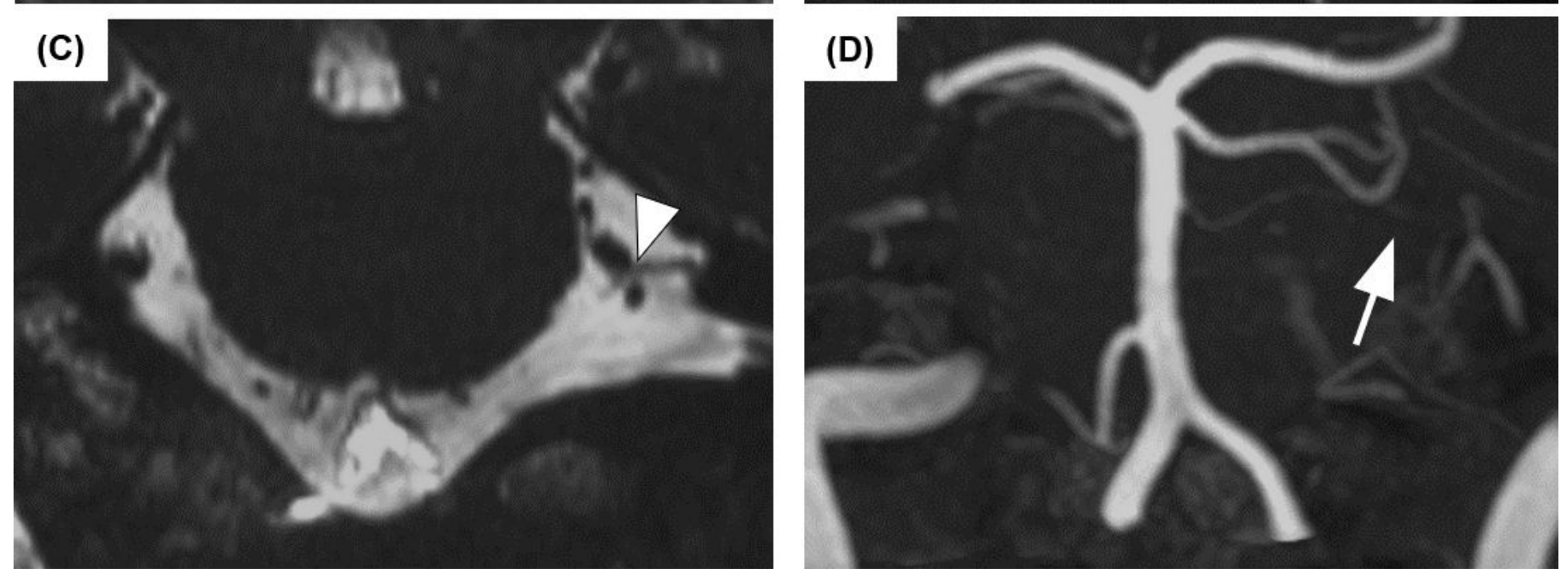

\section{Figure 2}

A 57-year old male with NVC on the trigeminal nerves (arrowheads). The responsible blood vessel was anterior inferior cerebellar artery (arrow). Transverse view of 3D-CISS (A), sagittal view (B), coronal view (C), and MIP display of 3D-TOF MRA (D). 
The scores of Oral DRS

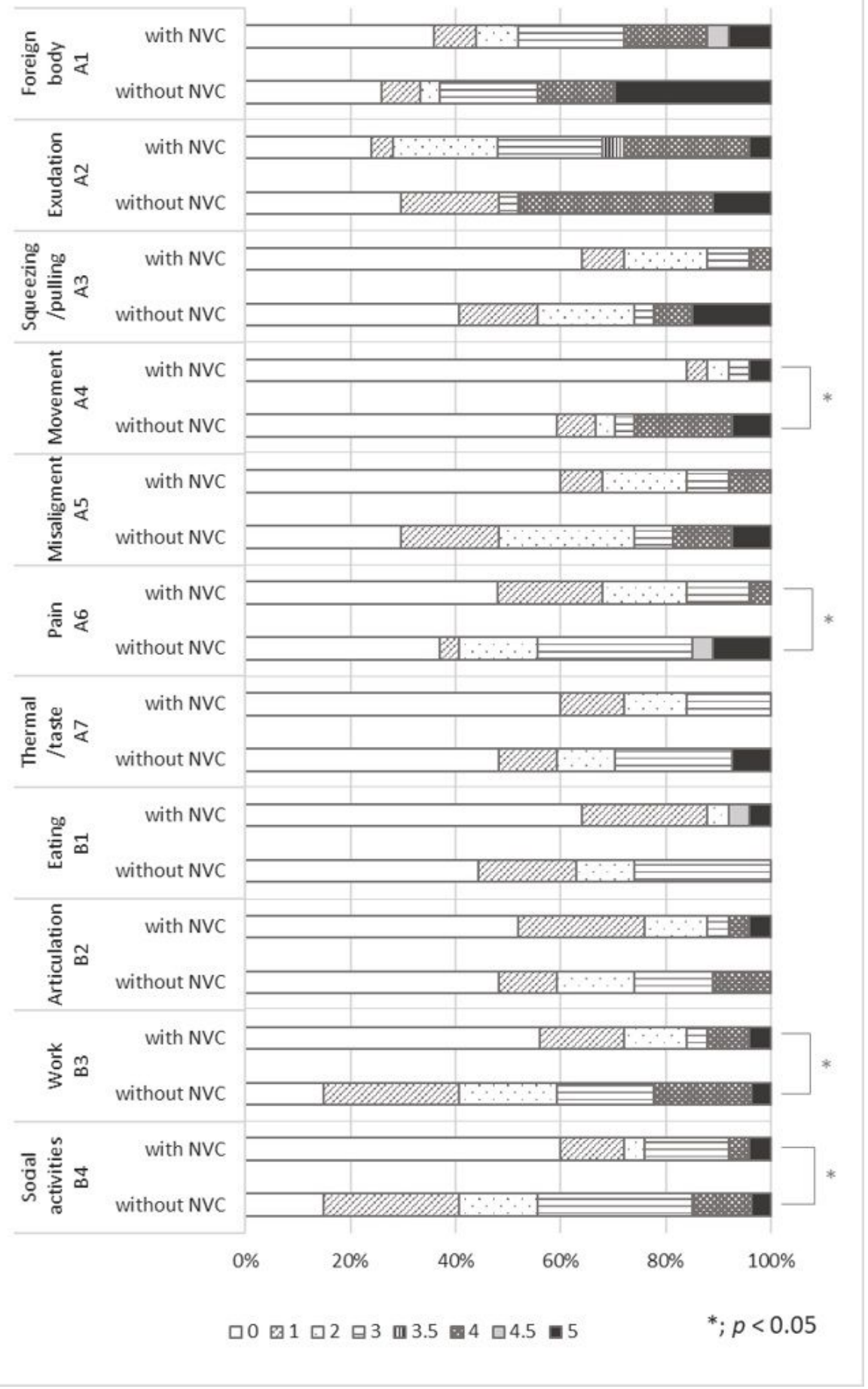

\section{Figure 3}

The scores of Oral DRS in the unilateral OC patients with and without NVC. The patients without NVC showed significantly higher scores in movement $(p=0.042)$, pain $(p=0.040)$, work $(p=0.013)$ and social activities ( $p=0.015)$, compared to the group with NVC. 